\title{
Representativeness of Offensive Scenarios to Evaluate Perceptual- Cognitive Expertise of Soccer Players
}

\author{
Filipe Casanova ${ }^{1, *}$, Júlio Garganta $^{1}$ and José Oliveira ${ }^{2}$ \\ ${ }^{I}$ Center of Research, Education, Innovation and Intervention in Sport (CIFI D), Faculty of Sport, University of Porto, \\ FADEUP, Portugal \\ ${ }^{2}$ Research Center in Physical Activity, Health and Leisure (CIAFEL), Faculty of Sport, University of Porto, FADEUP, \\ Portugal \\ ${ }^{3}$ Research Institute for Sport and Exercise Sciences, Liverpool John Moores University, Liverpool, UK
}

\begin{abstract}
In soccer, players have to carry out fast and accurate decisions in a complex and variable environment. The purpose of the present study was to set representative attacking sequences trials for further use in the research of perceptual-cognitive skills for playing soccer. Elite Portuguese soccer coaches $(n=4$, UEFA-A) were presented with separate test film sequences encompassing structured attacking soccer actions to determine the representativeness of the scenarios. In the experiment 41 offensive clips were viewed by the coaches. Each clip has approximately $5 \mathrm{~s}$ long with an inter-trial interval of $5 \mathrm{~s}$. To help the participants to the viewing process, just before the start of each clip a small circle surrounding the ball it is shown on screen to indicate the area of its first appearance. The order of presentation of video clips was counterbalanced and randomly determined, during both moments of evaluation. In all testing film sequences watched the representativeness of an attacking soccer phase was significantly concordant among the observers $(\mathrm{W}=1, p<0.05)$. The reliability between observers was statistically consistent $(\alpha=0.889)$. And the reproducibility of the results between both moments of evaluation was very high $(Z=0 ; p=1)$. The entire footage could be used in research that required knowing the tactical awareness of soccer players.
\end{abstract}

Keywords: Attacking Game Patterns, Reliability, Soccer.

\section{INTRODUCTION}

The fastest and the most accurate decisions of a soccer player elapses from information coming from several sources (i.e., the ball, the other players) and the decision-making process takes place under pressure with opponents trying to restrict the "time" and "space" available. Considering the specific constraints of training and competition demands, the performer has to carry out several tasks, such as: (i) to extract from a scene the essential information needed to predict future response requirements $[1,2]$; (ii) to recall and recognize patterns of play properly $[3,4]$; and (iii) to anticipate successfully the opponent's actions, based on advanced visual cues $[5,6]$. It has been hypothesized that superior performance in sport is based on perceptual and cognitive skill as well as the efficient and effective execution of movement patterns. To differentiate the perceptual-cognitive skills between participants, the researchers have used a range of perceptual and cognitive measures that could be demonstrative of the high-ability during a dynamical sport task, such as soccer [7].

\footnotetext{
*Address correspondence to this author at the Center of Research, Education, Innovation and Intervention in Sport (CIFI $\left.{ }^{2} \mathrm{D}\right)$, Faculty of Sport, University of Porto, FADEUP, Portugal; Tel: +351912787206;

E-mail: fcasanova@fade.up.pt
}

Ericsson and Smith [8] proposed a descriptive and inductive framework for the study of expertise which they referred to as the expert performance approach. Using this approach they identified three researching stages. The first necessitates that superior performance must be observed in situ and to design representative tasks such that reliable individual differences in performance can be objectively measured under laboratorial conditions. In the second stage the aim is to determine the mechanisms underlying performance using process-tracing measures such as eye-movement recordings, verbal protocol analysis and/or representative task manipulations. The final stage involves efforts to detail the adaptive learning and explicit acquisition processes relevant to the development of expertise, with potential implications for practice and instruction [9].

Williams et al. [7] have argued that perception and action are mutually interdependent, cyclical processes that directly constrain and influence one another. Although it has been well documented that the effective use of relevant advance visual cues facilitates sport performance by means of anticipating the intentions of the opponents [4, 7], the development of research protocols that provide relevant perception and action are warranted, as well as the several paradigms used to provide valuable insight into the effects that the decoupling of perception and action may have on performance $[7,9,10]$. 
Instead of using field-based conditions, some researchers have reported some limitations in using the video-based paradigms to capture the appropriate essence of superior performance [11]. However, when field-based approaches are not possible, presentation of video images are appropriate stimuli when compared to static slides. For example, Williams and Grant [12] have suggested a combination of subjective measures based on coach opinion and objective data based on qualitative and quantitative video analysis. They argued that in dynamic "open sports" the coaches' opinions could be gleaned pre- and post training using behavioral assessment scales $[13,14]$, while their validity could be substantiated using video analysis techniques [15]. Video analysis has already been used to measure anticipation skill in laboratory $[16,17]$ providing advantageously natural perception of the scene when compared with static slides [10].

Therefore, in the present study we aimed to create some game setting scenarios that could be representative of a real offensive soccer pattern. These situations were submitted to a panel of elite soccer coaches.

\section{METHODS}

\section{Participants}

The representativeness of the scenarios was determined through a panel of four elite Portuguese soccer coaches, with UEFA-A license and not less than 10 years experience of effective training. Participants were recruited and selected from the National Association of Portuguese Soccer Coaches' database. The study was carried out under the ethical guidelines of Faculty of Sport, University of Porto, and participants provided consent before taking part in the experiment.

\section{Match Scenarios}

Coaches were presented with separate video clips showing match sequences representing different game phases, i.e., attack, defense and transition play. The entire footage ends with an offensive skill that could unbalance the defensive organization. To guarantee that the scenario was truly realistic we conducted three practical sessions before the video recording. The first and the second experiment sessions were based on observing, memorizing and performing the theoretical schemes designed (for an example, see Fig. (1). The third session could be defined as a brief summary of the last two sessions. All the structured sequences were created by the Soccer Unit of Faculty of Sport, University of Porto. Portuguese Soccer players competing at the Second National League $(\mathrm{n}=22)$ participated in the scenarios build-up and permission was obtained from each one of them for public use of the recorded video images.

Each trial was filmed from the position behind $(15 \mathrm{~m})$ and slightly above $(5 \mathrm{~m})$ the goal with a 16 by 9 video camera (Sony DSR 570 DVCAM), such that the entire width of the playing field could be viewed and ensuring that potentially important information from wide positions was not eliminated. The elevated filming position helped give participants some element of depth. A single frame from a typical structured action sequence is depicted in Fig. (2).

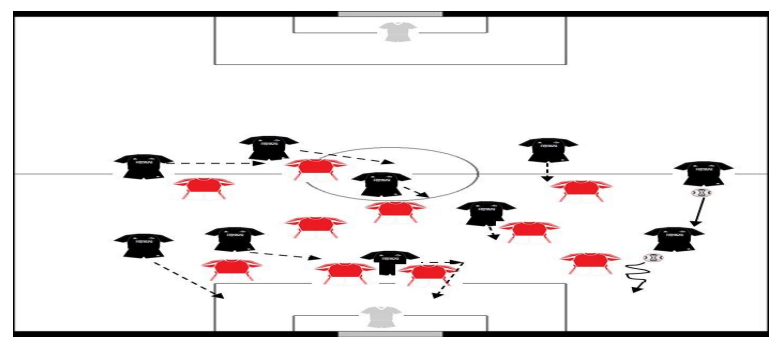

Fig. (1). Theoretical-scheme from the structured trial.

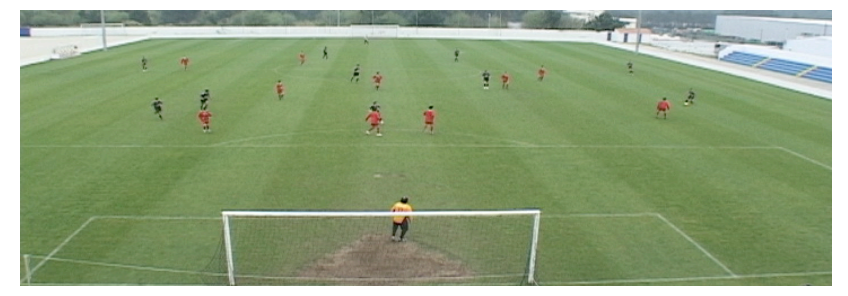

Fig. (2). Frame from the structured trial presented in video.

To edit the video into 41 different clips $^{1}$ a Pinnacle Software package software, Avid Liquid edition 7, was used. Each clip last approximately $5 \mathrm{~s}$ long with an inter-trial interval of $5 \mathrm{~s}$. The test consists in a clip-by-clip analysis, and just before the start of each clip, a small circle surrounding the ball appeared on screen to indicate the area of its first appearance. The clip stopped for $120 \mathrm{~ms}$ before the player in possession of the ball was about to make a pass or take a shot to goal or maintain the possession of the ball, and then the clip projection continued until the final event was finished, this last moment was identified when the screen turned to black. These three potential events were classed as offensive events: the Pass, i.e., a situation when the player has ball possession and attempts to play it to his team-mate with any part of the body except the head; the shot at goal, i.e., when the player is in ball possession and makes an attempt to score a goal for his team with any part of the body; the retain possession, i.e., when the player has ball possession and attempts to move with the ball, without losing it. All of the playing sequences finished when an attack to the goal is performed at the bottom of the screen.

\section{Procedures}

The video clips were presented in a dark room, in which the coaches were seated $2 \mathrm{~m}$ away from a Sony Television (model LCD KDL40P3600E). To ensure that the action was wholly perceived, the experts viewed the clips as many times as they wanted. Coaches answer using pencil and specific questionnaire. The panel of experts carried out another evaluation within 2 months of the first test.

The criterion was based on a 5-point Likert-type scale, where 5 means total agreement with the correct representativeness of the action, whereas 1 indicates total disagreement (see Table 1). Questionnaires based on Likert scales are often used in psychometrics, social studies and panels, in marketing research $[18,19]$, or in perceptual-cognitive performance research [20]. The order of presentation of video clips was counterbalanced and randomly determined, during

\footnotetext{
1 To have full access to the clips please send an email to fcasanova@fade.up.pt and/orjgargant@fade.up.pt
} 
Table 1. Likert-Type Scale (5 Point)

\begin{tabular}{|c|c|}
\hline & Likert-type Scale \\
\hline \hline 1 & Totally Disagree \\
\hline 2 & Disagree \\
\hline 3 & Neither Disagree nor Agree \\
\hline 4 & Agree \\
\hline 5 & Totally Agree \\
\hline
\end{tabular}

both moments of evaluation. Four additional trials were presented to participants prior to testing so that they could familiarize themselves with the video test and protocol.

\section{Statistical Analysis}

Descriptive statistical analysis was used to examine the valid values of the chosen Likert-point scale. To test the agreement between observers we used the Kendall's coefficient of concordance (W). Internal consistence reliability between observers was tested by using the Cronbach's Alpha $(\alpha)$. To test the construct validity (re-test) of the scenarios we used the nonparametric Wilcoxon Signed Rank Test (Z). Statistical significance was set at $p<0.05$ for all tests. The statistical software used was the SPSS Version 18.0 (SPSS Inc., Chicago, II).

\section{RESULTS}

The final offensive event of each clip is highlighted in Table 2.

The valid values of the chosen Likert-point scale shows that the entire sequence of the 41 clips projected was representative of a soccer game pattern, ending with an offensive event (see Table 3). As an exception, the clip 41 was rated at level 4 in the Likert-point Scale, since in the opinion of three expert coaches (A, C and D) this clip could be ended with a pass to another player.

In all test film sequences the representativeness of the game scenarios observed was significantly concordant among the observers $(\mathrm{W}=1 ; p<0.05)$. Moreover, the internal consistency reliability between observers showed that the responses scored were statistically consistent $(\alpha=0.889 ; p<$ $0.05)$.

Concerning the construct validity of the clips, the results obtained illustrated that when the experts watched again the projection of the 41 clips the values of the Likert-point scale were strongly reproduced $(Z=0 ; p=1)$.

\section{DISCUSSION}

The aim of the present study was to set representative attacking sequences trials for further use in the research of perceptual-cognitive skills for playing soccer. According to the results of the present study the panel of expert coaches agreed that the entire footage was representative of a real soccer situation which ends with a correct offensive event. Therefore, it seems useful tool to be used in perceptual- cognitive research, namely under controlled laboratory tasks. The design of the different game patterns used in this study was developed according to the three main categories of problems brought by team sports, which are: (i) space and time, (ii) information, and (iii) organization [21]. So, we have been constantly concerned with a tactical / strategic purpose, during the prescription of the game patterns and the practicing situations, as well.

Even the final event of the player in possession of the ball was sustained in Hughes et al. [22] reports. They defined a perturbation in soccer as an incident that changes the rhythmic flow of attacking and defending, leading to a shooting opportunity. For example, a perturbation could be identified from a penetrating pass, a dribble, a change of pace or any skill that creates a disruption in the defense and allows an attacker a shooting opportunity. In some cases, a perturbation of the defense may not result in a shot, owing to defensive skills or a lack of skill in attack. The clip stopped before the player in ball possession was either doing pass, shot at goal or retain possession. Both, pass and shot at goal have been associated with teams that has a higher percentage of success $[23,24]$ and the retain possession is being classified as the main goal to reach the truly purpose of the game, to score a goal. Bell-Walker et al. [24] reported that the successful teams at World Cup 2006, who were better able to hold ball possession, created more attempts at goal from open play and they also suggested that these teams had more positive attacking attitude.

Regarding to the duration of a video clip, McRobert et al. [25] noted that the perceptual and cognitive skills are inferred from the quality, speed and accuracy of an individual's performance, with minimal attempt to explain the cognitive processes involved during anticipation. Another scientific finding was reported by Ericsson and Simon [26] as they pointed out that subjects were able to recall accurately and completely the sequence of thoughts, cognitive information, after a $0.5-10 \mathrm{~s}$ task performance.

Although the video presentations reduce a threedimensional setting to a reality of two-dimensional scenarios, we tried to give to the subjects enough references of depth and width by elevating the film recording position and by using a 16 by 9 video camera, respectively. Another advantage of film projection is that it enables sequences of action to be reproduced in a consistent manner from trial to trial [27].

The results of the valid values of the Likert-point scale demonstrated that the panel of experts agreed with the representativeness of the clips. An exception of last decision of the player in ball possession was reported by three coaches in the clip number 41 , when the player did not pass the ball to a team-mate in a better position/space to receive it (see Table 2). Although being the game scenario included in clip 41 a common situation in soccer matches, the lower degree of total agreement between experts regarding the appropriate decision of the player in possession of the ball might preclude it's utilization as a scenario for assessment perceptualcognitive skill, since it could influence components such as advanced visual cue utilization, pattern recall and recogni tion, visual search behaviour and the knowledge of 
Table 2. Offensive Event in Each Clip

\begin{tabular}{|c|c|c|c|}
\hline Clip & \multicolumn{3}{|c|}{ Event } \\
\hline 2 & X (Left Winger) & & \\
\hline 3 & X (Center Midfielder) & & \\
\hline 5 & X (Center Midfielder) & & \\
\hline 6 & & & $\mathrm{X}$ \\
\hline 7 & & & $\mathrm{X}$ \\
\hline 8 & X (Left Winger) & & \\
\hline 11 & X (Striker) & & \\
\hline 12 & & & $\mathrm{X}$ \\
\hline 13 & & $\mathrm{X}$ & \\
\hline 14 & X (Left Midfielder) & & \\
\hline 15 & $\mathrm{X}$ (Striker) & & \\
\hline 16 & X (Left Midfielder) & & \\
\hline 17 & & $\mathrm{X}$ & \\
\hline 23 & & $\mathrm{X}$ & \\
\hline 24 & X (Left Midfielder) & & \\
\hline 25 & & $\mathrm{X}$ & \\
\hline 26 & X (Left Midfielder) & & \\
\hline 27 & & & $\mathrm{X}$ \\
\hline 28 & & & $\mathrm{X}$ \\
\hline 29 & X (Center Midfielder) & & \\
\hline 30 & & & $\mathrm{X}$ \\
\hline 31 & X (Right Winger) & & \\
\hline 32 & $\mathrm{X}$ (Striker) & & \\
\hline 33 & X (Center Midfielder) & & \\
\hline 34 & & & $\mathrm{X}$ \\
\hline 35 & X (Left Winger) & & \\
\hline
\end{tabular}


Table 2. Contd.....

\begin{tabular}{|c|c|c|c|}
\hline Clip & \multicolumn{3}{|c|}{ Event } \\
\hline 36 & X (Left Winger) & & \\
\hline 37 & X (Right Midfielder) & & \\
\hline 38 & X (Striker) & & \\
\hline 40 & X (Right Winger) & & \\
\hline 41 & X (Left Back) & & \\
\hline
\end{tabular}

Table 3. Mean Valid Values of the Likert-point Scale $( \pm$ SD) Pointed out by the Coaches, in Both Moments of Evaluation

\begin{tabular}{|c|c|c|}
\hline Coach & Test & Re-Test \\
\hline \hline A & $4.98 \pm 0.16$ & $4.98 \pm 0.16$ \\
\hline B & $5 \pm 0$ & $5 \pm 0$ \\
\hline C & $4.98 \pm 0.16$ & $4.98 \pm 0.16$ \\
\hline D & $4.98 \pm 0.16$ & $4.98 \pm 0.16$ \\
\hline
\end{tabular}

situational probabilities. In addition, previous published investigations support the use of this type of instrument to assess perceptual-cognitive performance [7, 10, 28-31].

Also, when presenting images with standard video, digital editing techniques can be used to add, remove, or distort normally invariant relations between different information sources. But to have not a detrimental effect on the perceptual information, we have to make sure that the manipulation or removal of perceptual information is only incremented in nonessential information. Ericsson [32] has argued that this type of method is particular relevant in sport where sequences of events are rarely if ever repeated in an exact form. Additionally, Ali [33] reported that using such instruments can enable scientists to carefully examine the core aspects of perceptual skill performance in soccer players.

\section{CONCLUSION}

Our findings indicated that the created scenarios representing soccer match patterns are a useful tool to evaluate perceptual-cognitive expertise, namely under controlled laboratory tasks. Moreover, more research is needed to understand the congruence between the results obtained in laboratory environment and performance of players in real game.

\section{CONFLICT OF INTEREST}

The authors confirm that this article content has no conflicts of interest.

\section{ACKNOWLEDGEMENT}

The lead author was funded by the Portuguese Foundation for Science and Technology (FCT) - Ministry of Science, Technology and Higher Education (MCTES; Ref. SFRH / BD / 36282 / 2007).

\section{REFERENCES}

[1] Abernethy B. Searching for the minimal essential information for skilled perception and action. Psychol Res 1993; 55: 131-8.

[2] Beek PJ, Jacobs D, Daffertshofer A, Huys R. Views on expert performance in sport from the joint perspectives of ecological psychology and dynamical systems theory. In: Starkes JL, Ericsson KA, Eds. Expert performance in sports: advances in research of sport expertise, Champaign, IL: Human Kinetics 2003; pp. 321-44.

[3] Allard F, Graham S, Paarsalu ML. Perception in sport: basketball. J Sport Psychol 1980; 2: 14-21.

[4] Williams AM, Davids K. Visual search strategy, selective attention, and expertise in soccer. Res Q Exerc Sport 1998; 69 (2): 111-28.

[5] Salmela JH, Fiorito P. Visual cues in ice hockey goaltending. Can J Appl Sports Sci 1979; 4 (1): 56-9.

[6] Williams AM, Burwitz L. Advance cue utilization in soccer. In: Reilly T, Clarys J, Stibbe A, Eds. Science and Football II. London: E \& FN Spon 1993; pp. 239-44.

[7] Williams AM, Davids K, Williams JG. Visual perception and action in sport. London: E \& FN Spon 1999.

[8] Ericsson KA, Smith J. Prospects and limits of the empirical study of expertise: an introduction. In: Ericsson KA, Smith J, Eds. Toward a general theory of expertise: prospects and limits. New York: Cambridge University Press 1991; pp. 1-18.

[9] Williams AM, Ericsson KA. Perceptual-cognitive expertise in sport: some considerations when applying the expert performance approach. Hum Mov Sci 2005; 24 (3): 283-307.

[10] Mann DTY, Williams AM, Ward P, Janelle CM. Perceptualcognitive expertise in sport: a meta-analysis. J Sport Exerc Psychol 2007; 29: 457-78.

[11] Abernethy B, Thomas KT, Thomas JT. Strategies for improving understanding of motor expertise (or mistakes) we made things we have learned. In: Starkes JL, Allard F, Ed. Cognitive issues in motor expertise. Amsterdam: Elsevier Science 1993; pp. 317-56.

[12] Williams AM, Grant A. Training perceptual skill in sport. Int J Sport Psychol 1999; 30: 194-220.

[13] French KE, Thomas JR. The relation of knowledge development to children's basketball performance. J Sport Psychol 1987; 9: 15-32.

[14] Oslin JL, Mitchell SA, Griffin LL. The game performance assessment instrument (GPAI): development and preliminary validation. J Teach Phys Educ 1998; 17: 231-41.

[15] Hughes M, Franks I. Notational analysis of sport. London: E. \& F. N. Spon 1997.

[16] Williams AM, Elliott D. Anxiety and visual search strategy in karate. J Sport Exerc Psychol 1999; 21: 362-75. 
[17] Williams AM, Ward P, Chapman C. Training perceptual skill in field hockey: is there transfer from the laboratory to the field? Res Q Exerc Sport 2003; 74 (1): 98-103.

[18] Hartley SL, MacLean WE Jr. A review of the reliability and validity of Likert-type scales for people with intellectual disability. J Intellect Disabil Res 2006; 50 (part II): 813-27.

[19] Dawes J. Do data characteristics change according to the number of scale points used? An experiment using 5-point, 7-point and 10point scales. Int J Mark Res 2008; 50 (1): 61-77.

[20] Williams AM, Hodges NJ, North JS, Barton G. Perceiving patterns of play in dynamic sport tasks: investigating the essential information underlying skilled performance. Perception 2006; 35: 317-32.

[21] Gréhaigne JF. A new method of goal analysis. Sci Football 1991; 5: 10-16.

[22] Hughes M, Dawkins N, David D, Mills J. The perturbation effect and goal opportunities in soccer. J Sports Sci 1998; 16: 20-1.

[23] Ali AH. In: Reilly T, Lees A, Davids K, Murphy WJ, Eds. Science and football: proceedings of the first world congress of science and football. London: E \& FN Spon 1988; pp. 302-9.

[24] Bell-Walker J, McRobert A, Ford P, Williams MA. Quantitative analysis of successful teams at the 2006 world cup finals. Insight $\mathbf{J}$ 2006; 36-43.

[25] McRobert AP, Williams AM, Ward P, Eccles DW. Tracing the process of expertise in a simulated anticipation task. Ergonomics 2009; 52 (4): 474-83.
[26] Ericsson KA, Simon HA. Protocol analysis: verbal reports as data United States of America: Massachusetts Institute of Technology 1999.

[27] Williams AM, Ward P. In: Tennenbaum G, Eklund R, Eds. Handbook of sport psychology. $3^{\text {rd }}$ ed. New York: John Wiley \& Sons 2007; pp. 203-23.

[28] Ericsson KA, Williams AM. Capturing naturally occurring superior performance in the laboratory: translational research on expert performance. J Exp Psychol Appl 2007; 13 (3): 115-23.

[29] Williams AM, Ford PR. Expertise and expert performance in sport. Int Rev Sport Exerc Psychol 2008; 1(1): 4-18.

[30] North JS, Williams AM. Identifying the critical time period for information extraction when recognizing sequences of play. Res $Q$ Exerc Sport 2008; 79 (2): 268-73.

[31] Casanova F, Oliveira J, Williams AM, Garganta J. Expertise and perceptual-cognitive performance in soccer: a review. Port J Sports Sci 2009; 9(1): 115-22.

[32] Ericsson KA. Expert performance in sports: advances in research on sport expertise. In: Starkes JL, Ericsson KA, Eds. Champaign, Illinois: Human Kinetics 2003; pp. 371-402.

[33] Ali A. Measuring soccer skill performance: a review. Scand J Med Sci Sports 2011;21: 170-83.

Received: November 08, 2011

Revised: June 19, 2012

Accepted: June 21, 2012

(C) Casanova et al.; Licensee Bentham Open.

This is an open access article licensed under the terms of the Creative Commons Attribution Non-Commercial License (http://creativecommons.org/licenses/ by-nc/3.0/) which permits unrestricted, non-commercial use, distribution and reproduction in any medium, provided the work is properly cited. 